\title{
Recommendations on Management of Locally Advanced Rectal Cancer During the COVID-19 Pandemic: an Iranian Consensus
}

\author{
Zahra Siavashpour $^{1} \cdot$ Farzad Taghizadeh-Hesary ${ }^{1} \cdot$ Afshin Rakhsha ${ }^{1}$ \\ Published online: 13 July 2020 \\ (C) Springer Science+Business Media, LLC, part of Springer Nature 2020
}

\begin{abstract}
Purpose Today, the rapid outbreak of COVID-19 is the leading health issue. Patients with cancer are at high risk for the development of morbidities of COVID-19. Hence, oncology centers need to provide organ-based recommendations for optimal management of cancer in the COVID-19 era.

Methods In this article, we have provided the recommendations on management of locally advanced rectal cancer during the COVID-19 pandemic based on our experience in Shohada-e Tajrish Hospital, Iran.

Results We recommend that patients with locally advanced rectal cancer should be managed in an individualized manner in combination with local conditions related to COVID-19.

Conclusion Our recommendation may provide a guide for oncology centers of developing countries for better management of locally advanced rectal cancer.
\end{abstract}

Keywords COVID-19 $\cdot$ Rectal cancer $\cdot$ Chemotherapy $\cdot$ Radiotherapy

\section{Introduction}

Since March 11, 2020, wherein the World Health Organization declared the novel coronavirus disease (COVID-19) a pandemic, healthcare providers around the world developed guidelines on the management of individuals with underlying diseases, including cancer [1-5]. Given the immune-compromised status of patients with cancer, they are susceptible to higher morbidity and mortality during COVID19 pandemic $[6,7]$. To reduce the exposure of patients with cancer during the era of COVID-19, several recommendations are presented as guidance for prioritizing the treatment plan for subsets of patients. According to these guidelines, clinicians should consider chance of cure, chance of palliation of

Afshin Rakhsha

Yasamin98522@gmail.com

Zahra Siavashpour

Z_siavashpour@sbmu.ac.ir

Farzad Taghizadeh-Hesary

f_taghizadeh@sbmu.ac.ir

1 Department of Radiation Oncology, Shohada-e Tajrish Educational Hospital, Shahid Beheshti University of Medical Sciences, Tehran, Iran symptoms, risk of tumor local recurrence, tumor biology, and its proliferating behaviors $[8,9]$. Rectal cancer is an emerging healthcare issue among the Asian countries. As the third most lethal cancer, it causes about $8 \%$ of all cancer mortalities [10, 11]. Standard management of locally advanced rectal cancer (LARC) is a multidisciplinary approach to achieve optimal outcomes by reducing recurrence rate and preserving the anal sphincter. In the following sections, first, we have provided the common practice followed by our considerations during the COVID-19 era for the staging and management of LARC based on the usual approach of neoadjuvant, surgical, and adjuvant phase. Thereafter, we present the consensus of Shohada-e Tajrish Hospital for the management of LARC during the COVID-19 pandemic.

\section{Common Practice and Special Considerations During COVID-19}

\section{Diagnostic and Staging Workup}

Considering the limitation of widespread coronavirus testing and low sensitivity in asymptomatic patients, every patient with cancer must be assumed to be the carrier of SARSCoV-2 during the pandemic [9]. Therefore, several 
considerations must be addressed during the staging workup of patients with LARC over the COVID-19 era. Considering the possible existence of SARS-CoV-2 in the digestive tract [12], effective personnel protective equipment is needed for the digital rectal examination of patients. For the locoregional staging, pelvic magnetic resonance imaging (MRI) is superior to transrectal endoscopic ultrasound, as the latter is in direct contact with the gastrointestinal secretions. For the systemic staging, obtaining abdominal MRI concurrent with pelvic MRI seems to be an appropriate choice as it decreases the patients' trip. The instruments and surfaces of the endoscopic and imaging rooms must be strictly sterilized using specific disinfectants upon each examination, for example, by vaporized hydrogen peroxide for at least $2 \mathrm{~h}$ [13].

\section{Neoadjuvant Phase}

According to the American Joint Committee on Cancer (AJCC), LARCs are T3-4 and/or clinically node-positive tumors [11]. Given the high probability of residual after primary resection of LARC, the National Comprehensive Cancer Network (NCCN) recommends three approaches as the neoadjuvant options, including (a) neoadjuvant long-course RT (i.e., 45-50.4 Gy in 25-28 fractions) with concomitant fluoropyrimidine-based chemotherapy (LCCRT), with higher doses (i.e., up to 54 Gy) for unresectable tumors; (b) shortcourse hypo-fractionated RT (SCRT) (i.e., 25 Gy in 5 fractions); and (c) induction chemotherapy followed by chemoradiation, known as total neoadjuvant therapy (TNT). The third approach was added to the 2015 version of the NCCN guideline as an acceptable approach. The advantages of LCCRT are more downstaging of the tumor and sphincter preservation and less toxicity. The advantages of SCRT are its simplicity, no chemotherapy toxicities, and cost-effectiveness. The advantages of TNT are early eradication of micrometastasis, a higher rate of pathologic complete response (pCR), decrease in time of surgery, and improvement of chemotherapy completion [14-16]. The SCRT is a non-inferior alternative to LCCRT, in terms of locoregional recurrence, distant recurrence, and overall survival $[1,10,15,17]$. The value of LCCRT over SCRT is questioned during the pandemic for its increased risk of myelosuppression and infection. Moreover, SCRT minimizes patients' exposure to medical surroundings [17]. Therefore, during the COVID-19 outbreak, considering SCRT would be an appropriate choice for the neoadjuvant chemoradiation of LARC. If LCCRT was selected, capecitabine is recommended as an alternative to intravenous fluorouracil (5-FU) to decrease patients' exposure. While the data is still evolving, TNT may be considered an appropriate choice during COVID-19 due to the facilitation and delay of surgery. The Society of Surgical Oncology (SSO) prioritizes the TNT over others and selects the SCRT as the radiotherapy phase of TNT [18]. According to the phase 2 trials, two regimens are available as the induction chemotherapy of TNT, the CAPOX (capecitabine and oxaliplatin) and FOLFOX regimen (fluorouracil, leucovorin, and oxaliplatin) $[19,20]$. During COVID-19 pandemic, the CAPOX regimen is recommended as the induction chemotherapy of TNT to decrease the patients' exposure. According to the European Society for Medical Oncology (ESMO) guideline, several clinical settings are in priority for RT during the COVID-19 pandemic, including patients who developed severe complications due to disease progression (e.g., compressive organ failure and massive bleeding) [21]. In summary, the most accepted neoadjuvant therapy for patients with LARC is LCCRT. However, in the coronavirus outbreak, the definition of "standard of care" was changed fundamentally in many oncology centers around the world. Lowering the frequency and duration of the patients' exposure is the basis for the management of immunosuppressed patients with cancer [8]. An additional 10-20 Gy using intraoperative RT (IORT) or brachytherapy (BT) boost is recommended in cases of T4, recurrence tumors, or close/positive margins [22]. The available choices for boost RT following EBRT of rectal cancer are IORT, BT, and contact therapy. The IORT may not be an applicable choice during the pandemic due to the limitations of operations during the COVID-19 era. Endorectal BT is applicable because it does not need general anesthesia. The accessibility of contact X-ray BT (Papillon technique) is not as endorectal BT but it is an appropriate alternative for outpatient setting [24]. Optimizing the pathologic complete response with $\mathrm{R} 0$ resection is reported on the implementation of BT boost following EBRT [23, 24]. Consolidative chemotherapy after SCRT may improve the results. In 2014, Glynne-Jones et al. have shown the positive effect of four courses of FOLFOX regimen on downstaging of tumor administered as a consolidative chemotherapy after SCRT [25]. During the COVID-19 outbreak, CAPOX regimen looks like a better choice as consolidative chemotherapy to decrease patients' stay in the hospital. In March 2020, Simcock et al. proposed the delay in radiotherapy in specific situations during the COVID-19 outbreak [26]. However, this approach is not recommended for patients with LARC. Considering the natural history of LARC, delay in neoadjuvant chemoradiation may switch the non-surgical situation to an emergent situation through rectal obstructing or perforation. Therefore, timely management of LARC should be considered.

\section{Surgical Phase}

The longer mean operative time of proctectomy encounters the patients with rectal cancer to further risk to develop morbidities and mortalities of COVID-19 [27]. Notably, due to the putative spread of SARS-CoV-2 via carbon dioxide aerosolization, the consensus-based guideline from the SSO recommends evaluating the risk-to-benefit ratio before application 
of laparoscopic procedures [18]. To reduce the risk of infection, the rate of elective surgeries has been reduced during the COVID-19 outbreak. The ESMO prioritizes several clinical settings for surgical management of patients with rectal cancer, including emergent surgical circumstances (e.g., hollow viscus perforation, obstruction, peritonitis, massive gastrointestinal bleeding), post-surgical complications (e.g., perforation, anastomotic leak), and bone fracture due to metastasis [21]. If surgery was selected upon the neoadjuvant treatment, it should be delayed no longer than 12 weeks. This may increase the radiation-associated fibrosis that led to technically more difficult operations [28]. During the surge times, delayed operation at 6-8 weeks after SCRT seems to be an appropriate choice [19]. This approach improves the tumor shrinkage and facilitates the surgery and, therefore, decreases the rate of postoperative complications and hospitalization time. Notwithstanding the lack of clinical trial evidence, "watch and wait" may potentially be an available approach for a subset of patients with clinical complete response (cCR) to neoadjuvant therapy during COVID-19. There is not yet adequate evidence to recommend a "watch and wait" approach over surgery, but a non-surgical option may likely be available to patients in the future.

\section{Adjuvant Phase}

The benefit of adjuvant oxaliplatin-based over fluorouracilbased regimens for rectal cancers is still controversial [29]. Only one out of eight available clinical trials demonstrated the superiority of FOLFOX over 5-FU and leucovorin in terms of disease-free survival in patients with LARC who received preoperative CRT and TME [29]. Therefore, it is advised to choose fluoropyrimidine-based chemotherapy as a postoperative chemotherapy of patients with LARC to decrease the hematologic toxicities during the COVID-19 pandemic. If an oxaliplatin-based regimen was the choice, the FOLFOX and CAPOX are the two available regimens, extrapolating from clinical trials of colon cancer [30]. In this regard, CAPOX is considered more appropriate during the viral pandemic for minimizing patients' exposure.

\section{Special Populations}

Elderly patients need special attention in the era of COVID-19. Several studies have presented recommendations for better management of elderly patients with cancer during the COVID-19 outbreak. They concluded that BT is a feasible alternative to surgery in elderly patients upon well downstaging of the tumor by neoadjuvant (chemo)radiation [31]. The criteria of frail elderly patients according to comprehensive geriatric assessment (CGA) are dependence in at least one activity of daily living; presence of at least three grade 3 , or at least one grade 4 comorbidity (based on CIRS-G); or presence of at least one geriatric syndrome.
Guidelines recommend SCRT plus BT for frail elderly patients. Patients with comorbidities or older than 80 years need further attention due to the five times higher risk of death [31, 32]. Eventually, frail unresectable cases were considered a separate categorization using those algorithms. BT boost following EBRT may substitute surgery for frail elderly patients and in cases of distal or inoperable tumors $[25,31,32]$. Notably, in these cases, delay in BT up to 6-8 weeks after EBRT provides more downstaging of tumor that optimizes dose delivery and decreases the toxicity [25].

\section{The Consensus from the Shohada-e Tajrish Hospital}

Considering the aforementioned guidelines, a consensus is proposed for management of patients with LARC during the coronavirus pandemic. Scheduled treatment is varied based on the clinical stage, distance from mesorectal fascia (MRF-D), possibility of resection, and performance status.

For patients with $\mathrm{T} 1-2 \mathrm{~N}+/ \mathrm{T} 3 \mathrm{~N} \pm$ (with $>2 \mathrm{~mm}$ MRF-D) rectal cancer, SCRT is recommended. To obtain more clinical response, delayed surgery to 6-8 weeks after completion of SCRT is an appropriate option during the COVID-19 pandemic. Delayed surgery is non-inferior in terms of overall survival $[33,34]$. For patients with $\mathrm{T} 3 \mathrm{~N} \pm$ (with $\leq 2 \mathrm{~mm}$ MRF-D)/T4 disease, we suggest the TNT approach including LCCRT for two reasons: TNT allows the surgery to be safely delayed and LCCRT enhances the clinical response. To minimize patients' exposure during the COVID-19 era, CAPOX is preferred over FOLFOX and capecitabine over intravenous 5-FU for adjuvant/induction and concomitant chemotherapy, respectively. Our choice for the duration of postoperative chemotherapy of patients with T1-2N1/T3N1 disease is 3 months, as it showed non-inferiority results in terms of the 3-year disease-free survival in comparison with 6 months of chemotherapy [35]. In other cases, the standard 6 months of adjuvant chemotherapy is chosen at our institution. Patients who are not candidates for surgery need higher radiation doses to provide more local control. Being such, we add BT with a dose of 10 20 Gy in 2-4 fractions upon SCRT. Thereafter, chemotherapy for 3-6 months is used for control of the systemic disease. Table 1 summarizes the strategy of clinicians of the Shohada-e Tajrish Hospital in the management of patients with LARC during the COVID-19 outbreak.

\section{Conclusion}

The current COVID-19 pandemic has affected the management of patients with cancer. Similar to the so-called emergency plan of radiotherapy departments for urgent situations, oncology centers need institutional guidelines for optimal 
Table 1 The consensus from the Shohada-e Tajrish Hospital for management of locally advanced rectal cancer during the COVID-19 era

\begin{tabular}{|c|c|c|c|c|}
\hline Clinical stage $^{\dagger}$ & EBRT & Chemotherapy & Brachytherapy & Delayed time to Surgery \\
\hline $\begin{array}{l}-\mathrm{T} 1-2 \mathrm{~N}+ \\
-\mathrm{T} 3 \mathrm{~N} \pm(>2 \mathrm{~mm} \text { MRF-D })\end{array}$ & SCRT & Post-surgery & - & 6-8 weeks after $\mathrm{SCRT}^{\S}$ \\
\hline $\begin{array}{l}-\mathrm{T} 3 \mathrm{~N} \pm(\leq 2 \mathrm{~mm} \text { MRF-D }) \\
-\mathrm{T} 4 \mathrm{~N} \pm\end{array}$ & LCCRT & As induction and concomitant with RT & - & 6-8 weeks after TNT \\
\hline $\begin{array}{l}\text { - Unresectable } \\
\text { - Medically inoperable } \\
\text { - Frail elderly }\end{array}$ & SCRT & Post-RT & $10-20 \mathrm{~Gy} / 2-4$ fraction & - \\
\hline
\end{tabular}

$E B R T$ external beam radiotherapy, $L C C R T$ long-course chemoradiotherapy, $M R F-D$ distance from mesorectal fascia, $R T$ radiotherapy, $S C R T$ shortcourse radiotherapy, $T N T$ total neoadjuvant therapy

$\dagger$ Based on the American Joint Committee on Cancer (AJCC) staging

\$Daley time for downstaging (for T3-4 cases)

$\S$ According to the Latkauskas et al.'s trial

management of patients during the COVID-19 pandemic. This article presented the consensus of the Clinical Oncology department of Shohada-e Tajrish Hospital for better management of patients with locally advanced rectal cancer during the COVID-19 pandemic. The recommendations discussed here were developed based on our experience in the most advanced oncology center in Iran. During COVID19, individualized management of LARC should be considered. Priority should always be given to the life-threatening conditions. We hope that these recommendations could provide reasonable references for oncologists worldwide facing the COVID-19 pandemic.

Acknowledgments The authors would like to express their gratitude to the staffs of Shohada-e Tajrish Hospitals of Tehran, Iran. At the end, the authors would like to express their gratitude to all physicians and nurses all around the world who are doing their best efforts to treat patients suffering from COVID-19.

Authors' Contributions A.R. designed the research; A.R. and Z.S. acquired data; A.R., Z.S., and F.T.H. performed the research; Z.S. and F.T.H. wrote the paper. All authors gave final approval of the version to be submitted.

Data Availability Not applicable.

\section{Compliance with Ethical Standards}

Conflict of Interest The authors declare that they have no conflict of interest.

Code Availability Not applicable.

\section{References}

1. Marijnen CAM, Peters FP, Rodel C, Bujko K, Haustermans K, Fokas E, et al. International expert consensus statement regarding radiotherapy treatment options for rectal cancer during the COVID
19 pandemic. Radiother Oncol. 2020;148:213-5. https://doi.org/ 10.1016/j.radonc.2020.03.039.

2. Achard V, Tsoutsou P, Zilli T. Radiotherapy in the time of the coronavirus pandemic: when less is better. Int J Radiat Oncol Biol Phys. 2020;107:600-1. https://doi.org/10.1016/j.ijrobp.2020. 03.008.

3. Shankar A, Saini D, Roy S, Mosavi Jarrahi A, Chakraborty A, Bharti SJ, et al. Cancer care delivery challenges amidst coronavirus disease-19 (COVID-19) outbreak: specific precautions for cancer patients and cancer care providers to prevent spread. Asian Pac J Cancer Prev. 2020;21(3):569-73.

4. Taghizadeh-Hesary F, Akbari H. The powerful immune system against powerful COVID-19: a hypothesis. Med Hypotheses. 2020;22:109762. https://doi.org/10.1016/j.mehy.2020.109762.

5. Motlagh A, Yamrali M, Azghandi S, Azadeh P, Vaezi M, Ashrafi F, et al. COVID19 prevention \& care; a cancer specific guideline. Arch Iran Med. 2020;23(4):255-64.

6. Yu J, Ouyang W, Chua MLK, Xie C. SARS-CoV-2 transmission in patients with cancer at a tertiary care hospital in Wuhan, China. JAMA Oncol. 2020. https://doi.org/10.1001/jamaoncol.2020.0980.

7. Mehta V, Goel S, Kabarriti R, Cole D, Goldfinger M, AcunaVillaorduna A, et al. Case fatality rate of cancer patients with COVID-19 in a New York hospital system. Cancer Discov. 2020;10:935-41. https://doi.org/10.1158/2159-8290.CD-20-0516.

8. van de Haar J, Hoes LR, Coles CE, Seamon K, Frohling S, Jager D, et al. Caring for patients with cancer in the COVID-19 era. Nat Med. 2020;26(5):665-71. https://doi.org/10.1038/s41591-0200874-8.

9. Aminian A, Safari S, Razeghian-Jahromi A, Ghorbani M, Delaney CP. COVID-19 outbreak and surgical practice: unexpected fatality in perioperative period. Ann Surg. 2020;272:e27-9.

10. Nacion AJD, Park YY, Kim NK. Contemporary management of locally advanced rectal cancer: resolving issues, controversies and shifting paradigms. Chin J Cancer Res. 2018;30(1):131-46. https:// doi.org/10.21147/j.issn.1000-9604.2018.01.14.

11. Halperin EC, Wazer DE, Perez CA, Brady LW. Perez \& Brady's principles and practice of radiation oncology. 7 th ed: Wolters Kluwer; 2019. p. 4614-79.

12. Wu Y, Guo C, Tang L, Hong Z, Zhou J, Dong X, et al. Prolonged presence of SARS-CoV-2 viral RNA in faecal samples. Lancet Gastroenterol Hepatol. 2020;5(5):434-5. https://doi.org/10.1016/ s2468-1253(20)30083-2.

13. Ren X, Chen B, Hong Y, Liu W, Jiang Q, Yang J, et al. The challenges in colorectal cancer management during COVID-19 epidemic. Ann Transl Med. 2020;8(7):498. 
14. Ngan SY. Preoperative treatment of locally advanced rectal cancer: assets and drawbacks of short course and long course in clinical practice. Semin Radiat Oncol. 2016;26(3):186-92. https://doi.org/ 10.1016/j.semradonc.2016.02.007.

15. Raldow AC, Chen AB, Russell M, Lee PP, Hong TS, Ryan DP, et al. Cost-effectiveness of short-course radiation therapy vs longcourse chemoradiation for locally advanced rectal Cancer. JAMA Netw Open. 2019;2(4):e192249. https://doi.org/10.1001/ jamanetworkopen.2019.2249.

16. Leeds IL, Fang SH. Neoadjuvant therapy for rectal cancer. Dis Colon Rectum. 2018;61(8):883-6.

17. Romesser PB, Wu AJ, Cercek A, Smith JJ, Weiser M, Saltz L, et al. Management of locally advanced rectal cancer during the COVID19 pandemic: a necessary paradigm change at Memorial Sloan Kettering Cancer Center. Adv Radiat Oncol. 2020. https://doi.org/ 10.1016/j.adro.2020.04.011.

18. Society of Surgical Oncology (SSO) (2020) Resource for Management Options of Colorectal Cancer During COVID-19,. https://www.surgonc.org/wp-content/uploads/2020/04/ColorectalResource-during-COVID-19-4.6.20.pdf. Accessed April 6, 2020.

19. Fernández-Martos C, Pericay C, Aparicio J, Salud A, Safont M, Massuti B, et al. Phase II, randomized study of concomitant chemoradiotherapy followed by surgery and adjuvant capecitabine plus oxaliplatin (CAPOX) compared with induction CAPOX followed by concomitant chemoradiotherapy and surgery in magnetic resonance imaging-defined, locally advanced rectal cancer: grupo cáncer de recto 3 study. J Clin Oncol. 2010;28(5):859-65.

20. Marechal R, Vos B, Polus M, Delaunoit T, Peeters M, Demetter P, et al. Short course chemotherapy followed by concomitant chemoradiotherapy and surgery in locally advanced rectal cancer: a randomized multicentric phase II study. Ann Oncol. 2012;23(6):152530.

21. European Society for Medical Oncology (ESMO) (2020) management and treatment adapted recommendations in the COVID-19 era: colorectal cancer (CRC). https://www.esmo.org/guidelines/ cancer-patient-management-during-the-covid-19-pandemic/ gastrointestinal-cancers-colorectal-cancer-crc-in-the-covid-19-era. Accessed June 25, 2020,

22. Siegel RL, Miller KD, Goding Sauer A, Fedewa SA, Butterly LF, Anderson JC, et al. Colorectal cancer statistics, 2020. CA Cancer J Clin. 2020. https://doi.org/10.3322/caac.21601.

23. Omidvari S, Zohourinia S, Ansari M, Ghahramani L, ZareBandamiri M, Mosalaei A, et al. Efficacy and safety of low-doserate endorectal brachytherapy as a boost to neoadjuvant chemoradiation in the treatment of locally advanced distal rectal cancer: a phase-II clinical trial. Ann Coloproctol. 2015;31(4):123-30. https:// doi.org/10.3393/ac.2015.31.4.123.

24. Sun Myint A, Mukhopadhyay T, Ramani VS, Perkins K, Snee AJ, Jelley F, et al. Can increasing the dose of radiation by HDR brachytherapy boost following pre operative chemoradiotherapy for advanced rectal cancer improve surgical outcomes? Color Dis.
2010;12(Suppl 2):30-6. https://doi.org/10.1111/j.1463-1318.2010. 02322.x.

25. Glynne-Jones R, Tan D, Moran BJ, Goh V. How to select for preoperative short-course radiotherapy, while considering longcourse chemoradiotherapy or immediate surgery, and who benefits? Eur Oncol Haematol. 2014;10:17-24.

26. Simcock R, Thomas TV, Mercy CE, Filippi AR, Katz MA, Pereira IJ, et al. COVID-19: global radiation oncology's targeted response for pandemic preparedness. Clin Transl Radiat Oncol. 2020. https:// doi.org/10.1016/j.ctro.2020.03.009.

27. Skowron KB, Hurst RD, Umanskiy K, Hyman NH, Shogan BD. Caring for patients with rectal Cancer during the COVID-19 pandemic. J Gastrointest Surg. 2020;15:1. https://doi.org/10.1007/ s11605-020-04645-z.

28. Monson J, Weiser M, Buie W, Chang GJ, Rafferty J, Buie WD, et al. Practice parameters for the management of rectal cancer (revised). Dis Colon Rectum. 2013;56(5):535-50.

29. Hattori N, Nakayama G, Uehara K, Aiba T, Ishigure K, Sakamoto E, et al. Phase II study of capecitabine plus oxaliplatin (CapOX) as adjuvant chemotherapy for locally advanced rectal cancer (CORONA II). Int J Clin Oncol. 2020;25(1):118-25. https://doi. org/10.1007/s10147-019-01546-3.

30. Loree JM, Sha A, Soleimani M, Kennecke HF, Ho MY, Cheung WY, et al. Survival impact of CAPOX versus FOLFOX in the adjuvant treatment of stage III colon cancer. Clin Colorectal Cancer. 2018;17(2):156-63. https://doi.org/10.1016/j.clcc.2018. 01.010 .

31. Wang SJ, Hathout L, Malhotra U, Maloney-Patel N, Kilic S, Poplin E, et al. Decision-making strategy for rectal cancer management using radiation therapy for elderly or comorbid patients. Int $\mathrm{J}$ Radiat Oncol Biol Phys. 2018;100(4):926-44. https://doi.org/10. 1016/j.ijrobp.2017.12.261.

32. Policy Brief : The Impact of COVID-19 on older persons (2020) United Nation. https://unsdg.un.org/sites/default/files/2020-05/ Policy-Brief-The-Impact-of-COVID-19-on-Older-Persons.pdf. Accessed May 2020.

33. National Comprehensive Cancer Network (NCCN) (2020) Rectal cancer,. https://www.nccn.org/professionals/physician_gls/pdf/ rectal.pdf. Accessed June 25, 2020.

34. Latkauskas T, Pauzas H, Kairevice L, Petrauskas A, Saladzinskas Z, Janciauskiene R, et al. Preoperative conventional chemoradiotherapy versus short-course radiotherapy with delayed surgery for rectal cancer: results of a randomized controlled trial. BMC Cancer. 2016;16(1):1-7.

35. Grothey A, Sobrero AF, Shields AF, Yoshino T, Paul J, Taieb J, et al. Duration of adjuvant chemotherapy for stage III colon cancer. N Engl J Med. 2018;378(13):1177-88.

Publisher's Note Springer Nature remains neutral with regard to jurisdictional claims in published maps and institutional affiliations. 